\title{
Effect of Sepimostat Mesilate on the Development of Glomerulonephritis in NZB/W F1 Mice
}

\author{
Hiroshi Watanabe ${ }^{*}$, Shuzo Sato, Rie Saito, Haruyo Iwadate, Hiroko Kobayashi and Hiromasa Ohira \\ Department of Gastroenterology and Rheumatology, School of Medicine, Fukushima Medical University, Fukushima \\ 960-1295, Japan
}

\begin{abstract}
Objective: To determine whether sepimostat mesilate inhibits activation of the complement pathways, and to evaluate the effectiveness of sepimostat mesilate on the development of glomerulonephritis in NZB/W F1 mice.

Methods: We used the Wielisa complement functional kit to assess the inhibitory effect of sepimostat mesilate on activation of the complement pathways. Groups of $10 \mathrm{NZB/NZW} \mathrm{mice} \mathrm{(age} \mathrm{18-22} \mathrm{weeks)} \mathrm{were} \mathrm{given} \mathrm{sepimostat} \mathrm{mesilate}$ $(200 \mu \mathrm{g} /$ dose) or glucose (control) five times a week for 5 weeks after onset of proteinuria.

Results: Sepimostat mesilate dose-dependently inhibited the activity of all complement pathways. Administration of sepimostat mesilate after disease onset lowered the levels of blood urea nitrogen $(243.2 \pm 63.1$ versus $120.9 \pm 22.1 \mu \mathrm{g} / \mathrm{dl}$; $\mathrm{p}<0.0001), \mathrm{C} 4 \mathrm{~d}(0.244 \pm 0.083$ versus $0.153 \pm 0.059 \mathrm{ng} / \mathrm{dl} ; \mathrm{p}=0.011)$, and delayed the development of proteinuria $(0.822 \pm$ 0.116 versus $0.470 \pm 0.093 \mathrm{mg} / \mathrm{mouse} / \mathrm{day} ; \mathrm{p}=0.046$ ) at the end of treatment ( 22 weeks of age). After discontinuation of administration, blood urea nitrogen, $\mathrm{C} 4 \mathrm{~d}$ level, and proteinuria rapidly became elevated with no difference between the groups. Eventually, mortality was similar between treated and untreated mice.

Conclusions: Sepimostat mesilate could be a therapeutic option for lupus nephritis.
\end{abstract}

Keywords: Sepimostat mesilate, systemic lupus erythematosus, NZB/W F1 mouse.

\section{INTRODUCTION}

The complement system is intimately involved not only in host defenses against infectious diseases but also in the pathology of many autoimmune diseases such as systemic lupus erythematosus (SLE) [1]. Activation of the complement system is triggered by immune complexes (ICs) that are either deposited or formed in situ within tissues. The kidney is a major site of immune complex deposition and complement activation, and about half of all lupus patients will develop nephritis [2].

NZB/W F1 mice spontaneously develop antibodies against autoantigens such as DNA. These antibodies form ICs and are deposited in the glomeruli. The deposited ICs trigger activation of the complement system, which causes glomerulonephritis, vasculitis, and cellular infiltration in the interstitium of the kidney $[3,4]$. The clinical and immunopathological similarities to human SLE mean that the NZB/WF1 mouse model is commonly used for screening of drugs intended to treat human SLE [5-7].

Sepimostat mesilate (6-amidino-2-naphthyl[4-(4,5dihydro-1H-imidazol-2-yl)amino] benzoate dimethane sulfonate) is a protease inhibitor developed by Torii Pharmaceutical Co., Ltd. (Tokyo, Japan) [8]. It has inhibitory potencies against proteases including $\mathrm{C} 1 \mathrm{r}, \mathrm{C} 1 \mathrm{~s}$, Factor D, plasmin, kallikrein, thrombin, and trypsin.

*Address correspondence to this author at the Fukushima Medical University, Department of Gastroenterology and Rheumatology, School of Medicine, 1 Hikarigaoka, Fukushima 960-1295, Japan; Tel: +81-24-5471111; Fax: +81-24-547-2055; E-mail: chiehiro@fmu.ac.jp
Furthermore, sepimostat mesilate (sepimostat) has inhibitory effects on various immunological reactions; it prevents complement-mediated hemolysis in vitro and in vivo, and protects against Forssman shock in guinea pigs $[8,9]$. As described above, sepimostat prevents activation of the classical and alternative complement pathways by inhibiting C1r, C1s, and factor D. However, the effect of sepimostat on activation of the lectin pathway has not yet been studied.

In this study: 1) we determined whether sepimostat inhibits the activation of the complement lectin pathway using the Wielisa complement function kit, an enzyme immunoassay for the qualitative determination of functional classical, lectin, and alternative pathways in serum; and 2) we administered sepimostat to NZB/W F1 mice to examine whether this agent can attenuate the severity of immunocomplex-mediated glomerulonephritis by inhibiting activation of the complement system.

\section{MATERIALS AND METHODOLOGY}

Mice

Six-week-old NZB/NZW F1 mice were purchased from Japan SLC (Hamamatsu, Japan) and maintained in the animal facility of Fukushima Medical University under specific pathogen-free conditions. This study was approved by the Fukushima Medical University Animal Protocols Review Board.

Inhibition Assay of Complement Activity by Sepimostat Using the Wielisa Kit

The Wielisa kit (Wieslab AB, Lund, Sweden) is an enzyme immunoassay for the qualitative determination of 
functional classical, lectin, and alternative complement pathways in serum. To determine the ability of sepimostat to inhibit complement activity, the concentration of sepimostat was adjusted to $200,100,50$, or $25 \mu \mathrm{g} / \mathrm{ml}$ in normal mouse serum, and the complement functional activity in each pathway was measured in the serum according to the manufacturer's instructions.

\section{Administration of Sepimostat}

Baseline was defined as when urinary protein excretion in NZB/W F1 mice exceeded 2SD when compared with $\mathrm{Balb} / \mathrm{c}$ mice. This occurred when the mice were 17 weeks of age. Administration of sepimostat was started one week later, at 18 weeks of age. The mice were divided into two groups of 10 mice each. One group was the control group; mice in this group were injected intraperitoneally with $0.1 \mathrm{ml}$ of $5 \%$ glucose five times per week. The treatment group received $200 \mu \mathrm{g}$ of sepimostat intraperitoneally five times a week for 5 weeks, ending when the mice were 22 weeks of age. Blood samples were taken from the mice prior to treatment, at the end of treatment, and 2 weeks following treatment.

\section{Measurement of Blood Urea Nitrogen (BUN)}

BUN was measured by the urease/indophenol method $[10,11]$. The urease reagent was prepared by adding $50 \mathrm{mg}$ of jack bean urease (Wako, Osaka, Japan) to $200 \mathrm{mg}$ of disodium ethylenediaminetetraacetate (EDTA-2Na). This solution was diluted to $500 \mathrm{ml}$ in distilled water and adjusted to $\mathrm{pH} 7.0$ with $\mathrm{NaOH}$. The phenol color reagent was prepared by adding $5 \mathrm{~g}$ of phenol to $25 \mathrm{mg}$ of $\mathrm{Na} 2 \mathrm{Fe}(\mathrm{CN}) 5 \mathrm{NO}-2 \mathrm{H} 2 \mathrm{O}$. This solution was diluted to $500 \mathrm{ml}$ in distilled water. To prepare the alkaline hypochlorite solution, $2.5 \mathrm{~g}$ of $\mathrm{NaOH}$ was dissolved in distilled water. Then, $3 \mathrm{ml}$ of $10 \% \mathrm{NaOCl}$ was added and diluted to $500 \mathrm{ml}$ in distilled water. To measure BUN, $4 \mu \mathrm{l}$ of each serum sample and standard urea solution was incubated with $200 \mu \mathrm{l}$ of urease reagent at $37^{\circ} \mathrm{C}$ for $15 \mathrm{~min}$. Then, $400 \mu \mathrm{l}$ of phenol color reagent was added, followed by generous vortexing. After adding $400 \mu \mathrm{l}$ of alkaline hypochlorite solution, sample tubes were incubated at $37^{\circ} \mathrm{C}$ for a further $20 \mathrm{~min}$. The optical density at $560 \mathrm{~nm}$ was measured, and BUN levels were calculated using a standard curve.

\section{Measurement of iC3b, C4d, and Bb in Serum}

Serum levels of complement cleavage products iC $3 b$, $\mathrm{C} 4 \mathrm{~d}$, and $\mathrm{Bb}$ were measured with a MicroVue iC3b EIA kit, MicroVue C4d EIA kit, and MicroVue Bb Plus kit (Quidel, San Diego, CA) according to the manufacturer's instructions.

\section{Urinary Protein Excretion}

Mice were placed in metabolic cages for 24-h collection of urine. To prevent bacterial growth, antibiotics were added to collection vials. Urinary protein excretion was determined using the Bio-Rad protein assay kit (Hercules, CA) according to the manufacturer's instructions.

\section{Pathology}

At 44 weeks of age, the treatment and control mice were sacrificed and the kidneys were removed. They were fixed with formaldehyde, embedded in paraffin, and then sectioned before staining with hematoxylin and eosin $(\mathrm{H} \& \mathrm{E})$ and periodic acid-Schiff (PAS).

\section{Statistics}

Statistical analysis was performed using the KruskalWallis test. Survival statistics were analyzed using the Kaplan-Meier method.

\section{RESULTS}

\section{Inhibition of the Complement Activity In Vitro by Sepimostat}

As shown in Fig. (1), sepimostat at doses of 25, 50, 100, and $200 \mu \mathrm{g} / \mathrm{ml}$ suppressed the activity of the classical pathway, alternative pathway, and lectin pathway in normal mouse serum in a dose-dependent manner.

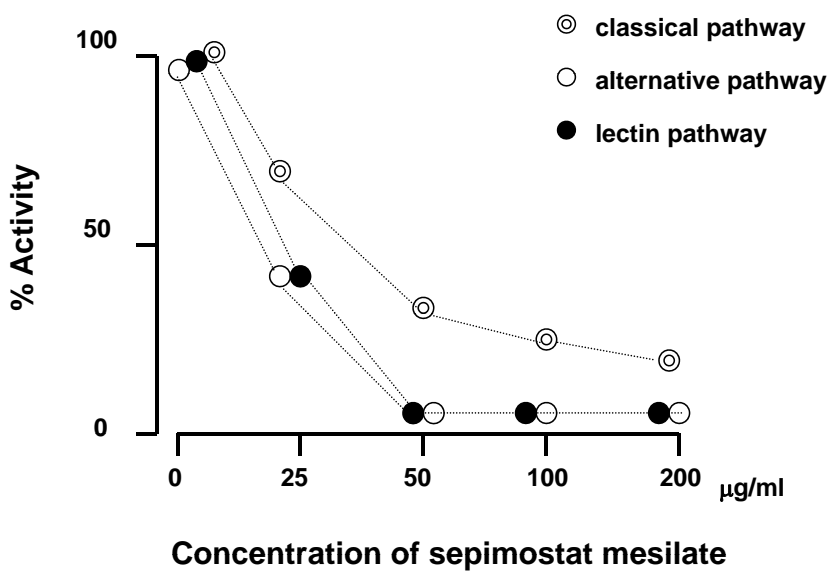

Fig. (1). Inhibitory effects on complement pathways. Suppression of complement function in the classical, alternative, and lectin pathways in normal mouse serum. The study revealed that sepimostat mesilate, at a concentration of $25 \mu \mathrm{g} / \mathrm{ml}$ and over, inhibited the function of all complement pathways in a dosedependent manner.

\section{BUN}

To investigate the effect of sepimostat on renal disease, serum BUN levels were measured. As shown in Fig. (2), serum BUN levels decreased in the treated group at the end of treatment ( 22 weeks) $(243.2 \pm 63.1$ versus $120.9 \pm 22.1$ $\mu \mathrm{g} / \mathrm{dl} ; \mathrm{p}<0.0001)$. However, by 24 weeks, serum BUN level had equalized in the two groups.

\section{Complement Cleavage Products iC3b, C4d, and Bb}

Measurement of complement cleavage products $\mathrm{C} 3 \mathrm{~d}$, $\mathrm{C} 4 \mathrm{~d}$, and $\mathrm{Bb}$ was performed at the following points: before treatment (16 weeks), at the end of treatment ( 22 weeks), and 2 weeks after treatment (24 weeks). As shown in Fig. (3), significant suppression of serum $\mathrm{C} 4 \mathrm{~d}$ was observed at the end of treatment ( 22 weeks) in the treated group $(0.244 \pm$ 0.083 versus $0.153 \pm 0.059 \mathrm{ng} / \mathrm{dl} ; \mathrm{p}=0.011)$. However, by 2 weeks after treatment, the differences were no longer significant between the control and treatment groups. There were no statistically significant differences in the serum concentrations of $\mathrm{iC} 3 \mathrm{~b}$ or $\mathrm{Bb}$ between the control group and the treated group at any time point (data not shown). 


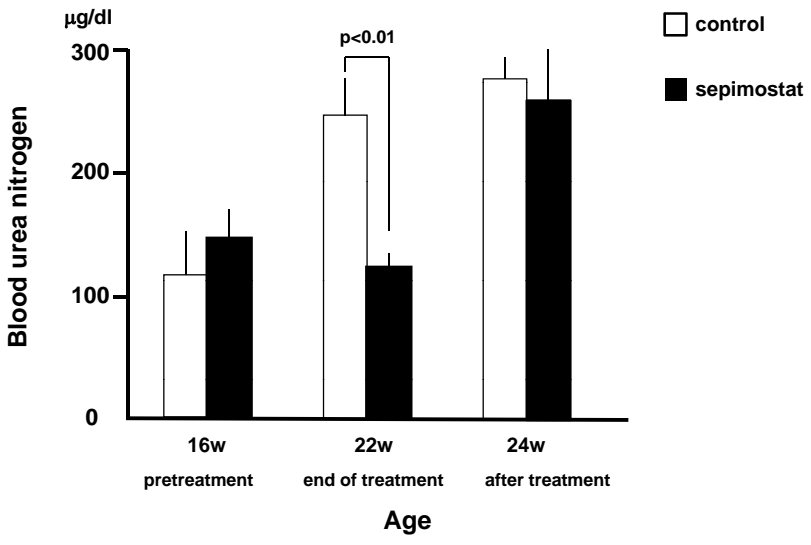

Fig. (2). Serum BUN levels in NZB/NZW F1 mice treated with sepimostat mesilate or glucose (controls). Data are expressed as the mean BUN level \pm SD in each group. A significantly reduced level of BUN was observed in sera from the sepimostat-treated group at the age of 22 weeks. By 2 weeks after treatment, the differences were no longer significant between the control and treatment groups. Data were analyzed using the Kruskal-Wallis test.

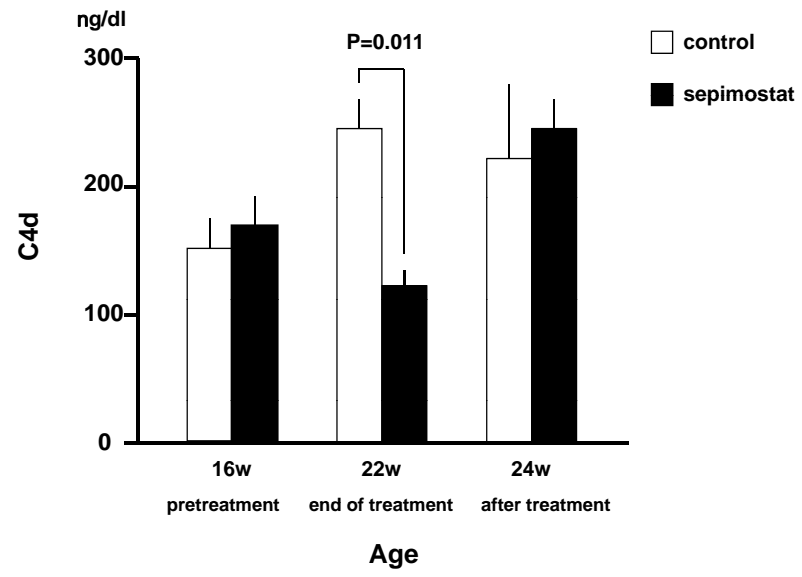

Fig. (3). Serum C4d levels in NZB/NZW F1 mice intraperitoneally injected with sepimostat mesilate or glucose (controls). Data are presented as the mean \pm SD in each group. Sera were obtained at the age of 16 weeks (pretreatment) and 22 weeks (end of treatment). A significant decrease in $\mathrm{C} 4 \mathrm{~d}$ level in the sepimostattreated group was observed at 22 weeks. By 2 weeks after treatment, the differences were no longer significant between the control and treatment groups.

\section{Urinary Protein Excretion}

Mice were placed in metabolic cages for $24-\mathrm{h}$ collection of urine, and we measured urine protein excretion once every 2 weeks. Fig. (4) shows the data before treatment (16 weeks), at the end of treatment ( 22 weeks) and 2 weeks after treatment ( 24 weeks). Proteinuria decreased in the treated group at the end of treatment $(0.822 \pm 0.116$ versus $0.470 \pm$ $0.093 \mathrm{mg} / \mathrm{mouse} / \mathrm{day} ; \mathrm{p}=0.046$ ). However, by 2 weeks after treatment, the differences were no longer significant between the control and treatment groups.

\section{Survival of Sepimostat-Treated Mice}

We did not observe prolonged survival in the treated group compared with the control group (Fig. 5).

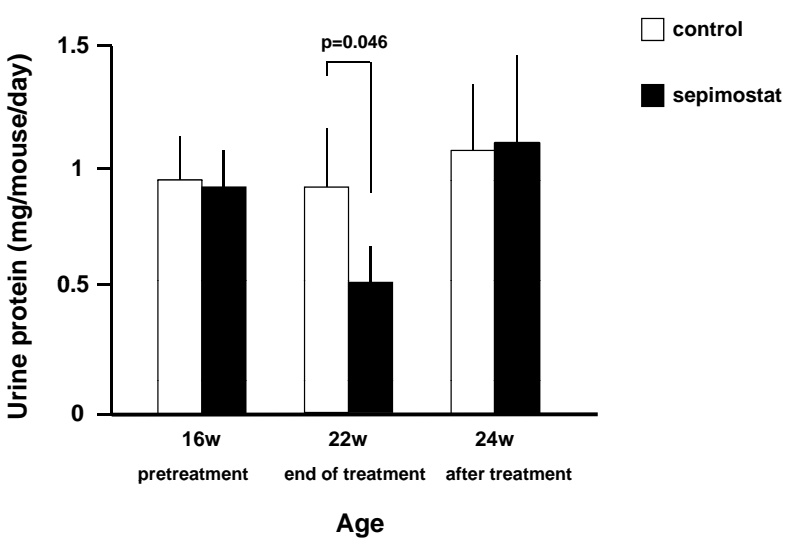

Fig. (4). Urinary protein excretion of NZB/NZW F1 mice. Data presented are the mean \pm SD of $24 \mathrm{~h}$ urinary protein excretion (mg/mouse/day) of six mice in each group. Each urine samples was tested twice for protein content. At the age of 22 weeks (end of treatment), decreased urine protein excretion was observed in the treated group. By 2 weeks after treatment, the differences were no longer significant between the control and treatment groups.

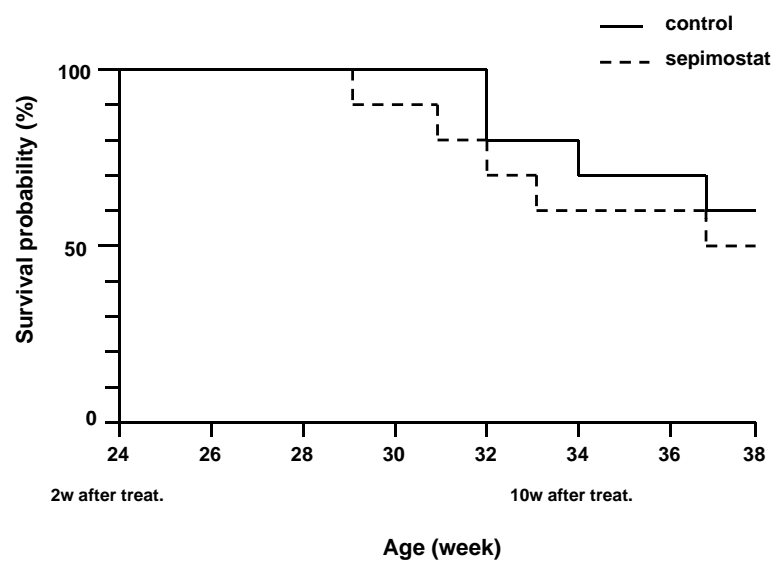

Fig. (5). Survival of NZB/NZW F1 mice. Mortality was similar in the sepimostat-treated mice and the control mice.

\section{Renal Pathology}

Mice surviving to 44 weeks of age were sacrificed, and the kidneys were removed for histopathological analysis. Renal findings in both the treatment and control groups included diffuse glomerular hypercellularity, wire loop lesions, mesangial expansion, and glomerular hyalinization (Fig. 6). No improvement in pathological features was detected in the treatment group.

\section{DISCUSSION}

In this study, we investigated the ability of sepimostat to inhibit complement activation using the Wielisa complement functional test, which assesses all three complement activation pathways individually. We observed that sepimostat dose-dependently inhibited the activity of all complement pathways, suggesting that it inhibits not only $\mathrm{C} 1 \mathrm{q}, \mathrm{C} 1 \mathrm{~s}$, and factor D as previously reported, but also other complement components that contribute to activation of the lectin pathway. If so, which component in the lectin pathway is inhibited by sepimostat? Mannose-binding lectinassociated serine protease (MASP) contributes to activation 

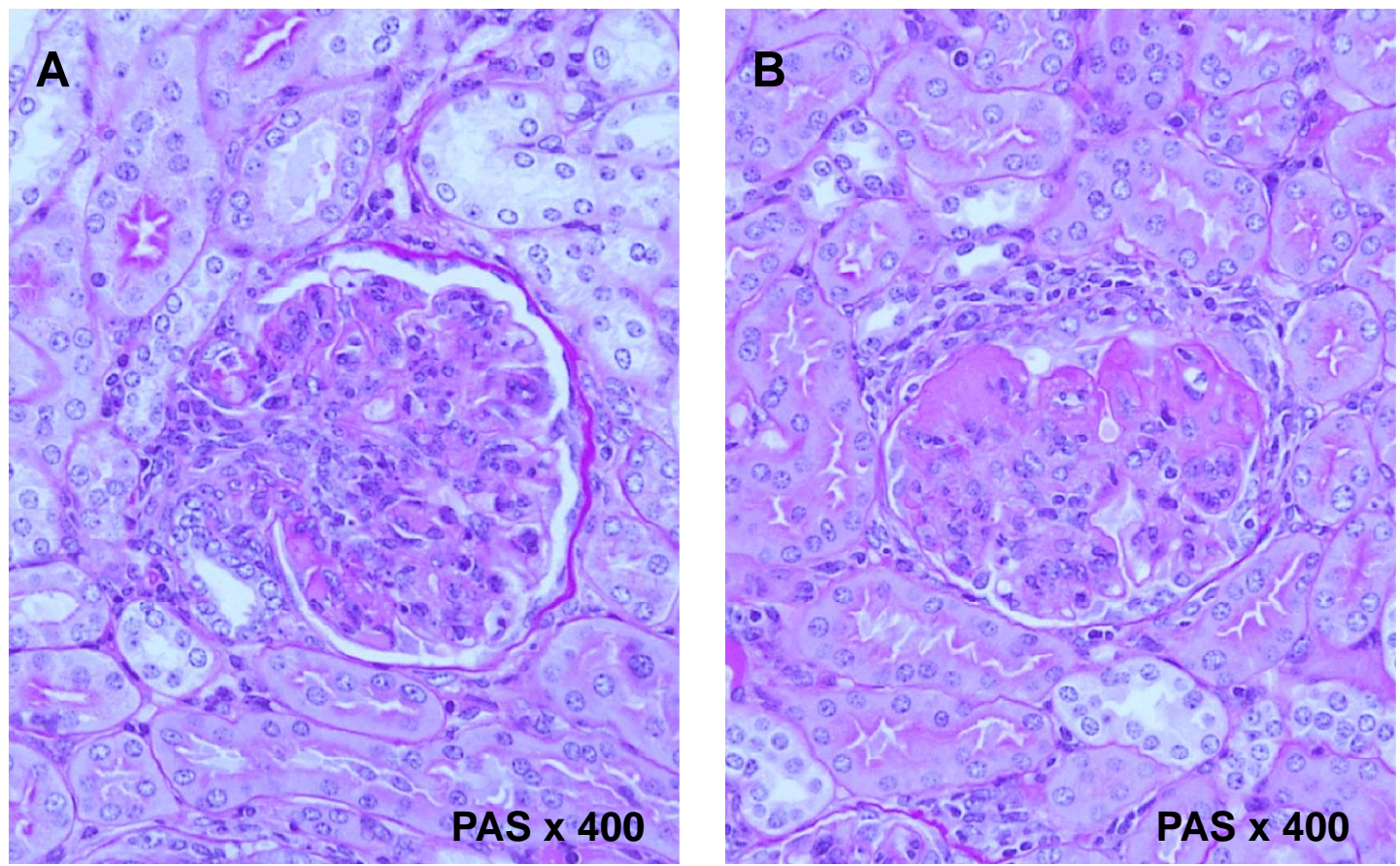

Fig. (6). Renal findings in sepimostat-treated mice (A) and control mice (B). In both groups, kidneys show diffuse glomerular hypercellularity, wire loop lesions, mesangial expansion, and glomerular hyalinization.

of the lectin pathway by binding mannose-binding lectin (MBL). The lectin complement pathway is closely related to the classical complement pathway in adaptive immunity, in terms of the structures as well as functions of their components. Both pathways are triggered by complexes consisting of collagenous proteins and serine proteases of the $\mathrm{MASP} / \mathrm{C} 1 \mathrm{r} / \mathrm{C} 1 \mathrm{~s}$ family [12-14]. Considering that MASP is a member of the same family as $\mathrm{C} 1 \mathrm{r}$ and $\mathrm{C} 1 \mathrm{~s}$, it is possible that sepimostat may inhibit the lectin pathway by inhibiting the potency of MASP.

We also observed that the administration of sepimostat after disease onset lowered the levels of BUN, C4d, and delayed the development of proteinuria. However, once the treatment was stopped, serum levels of BUN, C4d, and proteinuria became elevated with no difference between the groups. Eventually, mortality as well as histological damage were similar between treated and untreated mice.

Although it could not be proven by immunopathological methods, the decreased serum level of $\mathrm{C} 4 \mathrm{~d}$ in the sepimostattreated group suggests that sepimostat may alleviate glomerulonephritis in the NZB/w F1 mouse by inhibiting activation of the classical pathway, the lectin pathway, or both. Clearly, sepimostat does not have the function of inhibiting the formation of ICs as well as their deposition in the kidney. Therefore, the presumed mechanism is that, by inhibiting $\mathrm{C} 1 \mathrm{r}$ and $\mathrm{C} 1 \mathrm{~s}$, sepimostat inhibits the activation of the classical pathway triggered by deposition of ICs in the kidney, thereby alleviating inflammation and tissue damage. It seems likely that, because of the short half-life, the effects of sepimosat cease rapidly after it is stopped, and disease activity returns.

Sepimostat inhibits proteases other than those in the complement system $(\mathrm{C} 1 \mathrm{r}, \mathrm{C} 1 \mathrm{~s}$, Factor D), such as plasmin, kallikrein, thrombin, and trypsin. Therefore, it is possible that the reduction in proteinurea and serum BUN levels were caused not only by the inhibition of complement pathway activation, but also by the inhibition of these other serine proteases that may contribute to tissue damage in autoimmunity.

Inhibition of complement activation has been considered a potential therapeutic approach for SLE. However, the complement system appears to play a dual role in the progression of SLE; it has not only a pathogenic role in inducing local inflammation but also a beneficial role in the clearance of apoptotic cells and ICs [1]. Given that the complement system also has important physiological roles in host defense and immune homeostasis, potential risks are associated with systemic complement inhibition, particularly when prolonged treatment is required. Therefore, long-term administration of sepimostat could cause diseases (e.g., lupus-like disease, Neisseria infection) in patients with complement deficiency, due to the inhibition of the beneficial effects of complement; i.e., clearance of apoptotic cells and ICs, and host defense.

Several serine protease inhibitors (i.e., nafamostat mesilate, camostat mesilate, gabexate mesilate) with similar chemical structures to sepimostat are commonly used in pancreatitis and disseminated intravascular coagulation in Japan. Although these protease inhibitors strongly inhibit activation of the complement system, there have been no reports of adverse effects of opportunistic infections resulting from immune suppression. This may relate to the short half-life of these drugs (no more than 10 minutes). Furthermore, nafamostat mesilate has been reported to ameliorate glomerulonephritis in immunocomplex disease as well as in the NZB/W F1 mouse [15-17]. Therefore, it is possible that sepimostat could be a therapeutic option for lupus nephritis patients with steroid resistance or severe immune compromise. 
In conclusion, our study suggests that sepimostat may represent a novel treatment for immune-complex nephritis. Further long-term studies are needed to confirm the utility of this drug as a treatment for lupus nephritis.

\section{CONFLICT OF INTEREST}

The authors declare that we have no financial and personal relationships with other people or organizations that could inappropriately influence this work.

\section{REFERENCES}

[1] Bao L, Quigg RJ. Complement in lupus nephritis: the good, the bad, and the unknown. Semin Nephrol 2007; 27: 69-80.

[2] Balow JE, Boumpas DT, Austin III HA. In: Lahita RG, Ed. Systemic lupus erythematosus. San Diego: Academic Press 1999; pp. 657-85.

[3] Andrews BS, Eisenberg RA, Theoflopous AN, et al. Spontaneous murine lupus-like syndromes. Clinical and immunopathological manifestations in several strains. J Exp Med 1978; 148: 1198-215.

[4] Hahn B. In: Qallace HB, Ed. Dubois' lupus erythematosus. Baltimore: Williams and Wilkins 1997; pp. 339-47.

[5] Borel Y, Lewis RM, Andre-Schwatz J, Stollar BD, Diener E. Treatment of lupus nephritis in adult (NZB+NZW)F1 mice by cortisone-facilitated tolerance to nucleic acid antigens. J Clin Invest 1978; 61: 276-86

[6] Hahn BH, Knotts L, Hamilton TR. Influence of cyclophosphamide and other immunosuppressive drugs on immune disorders and neoplasia in NZB/NZW mice. Arthritis Rheum 1975; 18: 145-52.

[7] Schiffer L, Sinha J, Wang X, et al. Short term administration of costimulatory blockade and cyclophosphamide induces remission of systemic lupus erythematosus nephritis in NZB/W F1 mice by a mechanism downstream of renal immune complex deposition. $\mathrm{J}$ Immunol 1997; 71: 489-505.
[8] Oda M, Ino Y, Nakamura K, et al. Pharmacological studies on 6amidino-2-naphthyl [4-(4,5-dihydro-1H-imidazol-2-yl)amino]benzoate dimethane sulfonate (FUT-187). I: Inhibitory activities on various kinds of enzymes in vitro and anticomplement activity in vivo. Jpn J Pharmacol 1990; 52: 23-34.

[9] Nakamura K, Johmura A, Oda M, et al. Inhibitory effects of sepimostat mesilate (FUT-187) on the activities of trypsin-like serine proteases in vitro. Yakugaku Zasshi 1995; 115: 201-12. In Japanese.

[10] Kanai I. Blood urea nitrogen. In: Kanai M, Ed. Rinshoukensahouteiyou. Tokyo: Kanehara Shuppan 1983; pp. 42325. In Japanese.

[11] Kaplan A. In: Glick D, Ed. Methods of Biochemical Analysis. New York: John Wiley \& Sons 1954; pp. 311-24.

[12] Matsushita M, Fujita T. Activation of the classical complement pathway by mannose-binding protein in association with a novel C1s-like serine protease. J Exp Med 1992; 176: 1497-502.

[13] Thiel S, Vorup-Jansen T, Stover CM, et al. A second serine protease associated with mannan-binding lectin that activates complement. Nature 1997; 386: 506-10.

[14] Dahl MR, Thiel S, Matsusihta M, et al. MASP-3 and its association with distinct complexes of the mannan-binding lectin complement activation pathway. Immunity $2001 ; 15: 127-35$.

[15] Miyata T, Fujita Y, Inagi R, Inoue I, Sugiyama S, Maeda K. Effectiveness of nafamostat mesilate on glomerulonephritis in immune-complex diseases. Lancet 1993; 41: 1353.

[16] Fujita Y, Inoue I, Inagi R, et al. Inhibitory effect of FUT-175 on complement activation and its application for glomerulonephritis with hypocomplementemia. Nippon Jinzo Gakkai Shi 1993; 35 393-7. In Japanese.

[17] Ikehara S, Shimamura K, Aoyama T, Fujii S, Hamashima Y. Effect of FUT-175, a new synthetic protease inhibitor, on the development of lupus nephritis in (NZBx NZW) F1 mice. Immunology 1985; 55: 595-600. 